\title{
High Resolution Florida IR Silicon Immersion Grating Spectrometer and an M Dwarf Planet Survey
}

\author{
Jian Ge, Scott Powell, Bo Zhao, Ji Wang, Adam Fletcher, Sidney Schofield \& Jian Liu \\ Department of Astronomy, 211 Bryant Space Science Center, University of Florida \\ Email: jge@astro.ufl.edu \\ Tel: 352-294-1850 \\ Matthew Muterspaugh \\ Center of Excellence in Information System \& Department of Mathematical Sciences, College of \\ Engineering, Tennessee State University \\ Cullen Blake \\ Department of Astrophysical Sciences, Princeton University \\ Rory Barnes \\ Department of Astronomy, University of Washington
}

We report the system design and predicted performance of the Florida IR Silicon immersion grating spectromeTer (FIRST). This new generation cryogenic IR spectrograph offers broad-band high resolution IR spectroscopy with $\mathrm{R}=72,000$ at $1.4-1.8 \mu \mathrm{m}$ and $\mathrm{R}=60,000$ at $0.8-1.35 \mu \mathrm{m}$ in a single exposure with a $2 \mathrm{kx} 2 \mathrm{k}$ H2RG IR array. It is enabled by a compact design using an extremely high dispersion silicon immersion grating (SIG) and an R4 echelle with a $50 \mathrm{~mm}$ diameter pupil in combination with an Image Slicer. This instrument is operated in vacuum with temperature precisely controlled to reach long term stability for high precision radial velocity (RV) measurements of nearby stars, especially $M$ dwarfs and young stars. The primary technical goal is to reach better than $4 \mathrm{~m} / \mathrm{s}$ long term RV precision with $\mathrm{J}<9 \mathrm{M}$ dwarfs within 30 min exposures. This instrument is scheduled to be commissioned at the Tennessee State University (TSU) 2-m Automatic Spectroscopic Telescope (AST) at Fairborn Observatory in spring 2013. FIRST can also be used for observing transiting planets, young stellar objects (YSOs), magnetic fields, binaries, brown dwarfs (BDs), ISM and stars.

We plan to launch the FIRST NIR M dwarf planet survey in 2014 after FIRST is commissioned at the AST. This NIR M dwarf survey is the first large-scale NIR high precision Doppler survey dedicated to detecting and characterizing planets around 215 nearby $M$ dwarfs with $\mathrm{J}<10$. Our primary science goal is to look for habitable Super-Earths around the late M dwarfs and also to identify transiting systems for follow-up observations with JWST to measure the planetary atmospheric compositions and study their habitability. Our secondary science goal is to detect and characterize a large number of planets around $\mathrm{M}$ dwarfs to understand the statistics of planet populations around these low mass stars and constrain planet formation and evolution models. Our survey baseline is expected to detect $\sim 30$ exoplanets, including 10 Super Earths, within 100 day periods. About half of the Super-Earths are in their habitable zones and one of them may be a transiting planet. The AST, with its robotic control and ease of switching between instruments (in seconds), enables great flexibility and efficiency, and enables an optimal strategy, in terms of schedule and cadence, for this NIR M dwarf planet survey.

Key words: High resolution, silicon immersion grating, Doppler, infrared, exoplanets, spectrograph, M dwarfs \& habitable zones

Ground-based and Airborne Instrumentation for Astronomy IV, edited by lan S. McLean, Suzanne K. Ramsay, Hideki Takami, Proc. of SPIE Vol. 8446, 84463O - @ 2012 SPIE · CCC code: 0277-786X/12/\$18 · doi: 10.1117/12.925788 


\section{Introduction}

The discovery of more than 700 exoplanets over the past two decades has changed our view of the universe. The exoplanet worlds show great diversity, from giant planets very close to their host stars, to giant planets in extremely eccentric orbits, to multiple giant planets, to planets around binaries, to low mass planets as small as $\sim 2$ Earth masses. Recent advances in space-based photometric precision demonstrated by the NASA Kepler mission have helped to identify a few Earth-like planet candidates in the Habitable Zone (HZ) around solar type stars (Borucki et al. 2011). The ground-based optical radial velocity (RV) spectrograph, HARPS, at the European Southern Observatory 3.6 meter telescope, has detected two close-by, possibly habitable low mass planets, Gl 581d ( 7 Earth masses, Mayor et al. 2009) and HD85512b ( 3.6 Earth masses, Pepe et al. 2011), around low mass M dwarfs.

Although great progress has been made in exoplanet studies, one of the fundamental questions, "How common are Earth-like planets?" remains to be addressed. One efficient way to answer this question with currently available technology is to look where the signal of an Earth-like planet would be strongest---M dwarfs. Low-mass planets in the habitable zones of M-dwarfs yield larger and more frequent signals in both RVs and transits, and have a higher probability of transiting than similar objects around Sun-like stars. For this reason, dedicated searches for planets orbiting low-mass stars received strong support from the ExoPlanet Task Force and 2010 Decadal Survey.

Of particular interest are $\mathrm{M}$ dwarfs later than M4, where the mass, size, and temperature of the stars begin to rapidly decrease. To date, most exoplanet searches targeting $M$ dwarfs have been conducted at visible wavelengths (e.g., Endl et al. 2006; Johnson et al. 2007; Bonfils et al. 2011). Because the later type stars produce most of their light at redder wavelengths, only those earlier than M4 have been well studied. There are only 12 M4 or later type stars with V < 12 north of -30 degrees (Reid \& Gizis 1997). For comparison, there are about 300 nearby stars M4 or later with J $<9$ (Lepine \& Shara 2005). Therefore, for the latest types of stars, an observing program must operate where they emit the most light — near infrared (NIR).

The current state-of-the-art for NIR RV detection of planets around late M dwarfs has been demonstrated with the VLT's CRIRES $(\mathrm{R}=100,000)$ with moderate simultaneous wavelength coverage $\left(\begin{array}{ll}364 & \AA\end{array}\right)$ using an ammonia gas cell for calibration (Bean et al. 2010). Long-term ( $\sim 6$ months) RV precisions of 5 $\mathrm{m} / \mathrm{s}$ have been demonstrated with this system, indicating that high precision RV measurements can be achieved with IR Doppler instruments.

At the University of Florida, we are developing a new NIR cryogenic temperature controlled, high resolution, cross-dispersed echelle spectrograph dedicated for highprecision RV measurements.

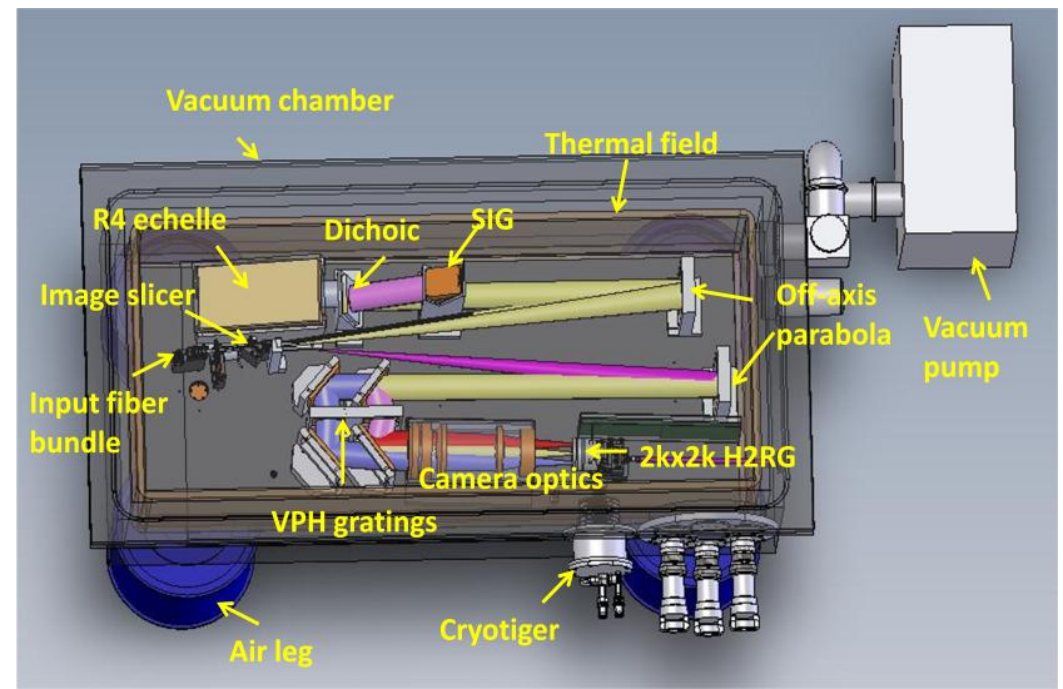

Figure 2.1. The overall optical/mechanical/cryogenic layout of the FIRST instrument system. The FIRST bench has $1.0 \times 0.5$ meter in dimension, a very compact design.

This instrument is called the Florida IR Silicon immersion grating spectromeTeter (FIRST). The primary 
technical goal is to reach better than $4 \mathrm{~m} / \mathrm{s}$ long term precision with $\mathrm{J}<9 \mathrm{M}$ dwarfs within 30 min exposures. It takes advantage of an emerging silicon immersion grating (SIG) technology and a matured $2 \mathrm{kx} 2 \mathrm{k}$ H2RG IR detector array to offer high resolution and large simultaneous wavelength coverage in a single exposure. This instrument is scheduled to be commissioned at the 2-m Automatic Spectroscopic Telescope (AST) (Eaton \& Williamson 2007) at Fairborn Observatory in spring 2013. In this paper, we
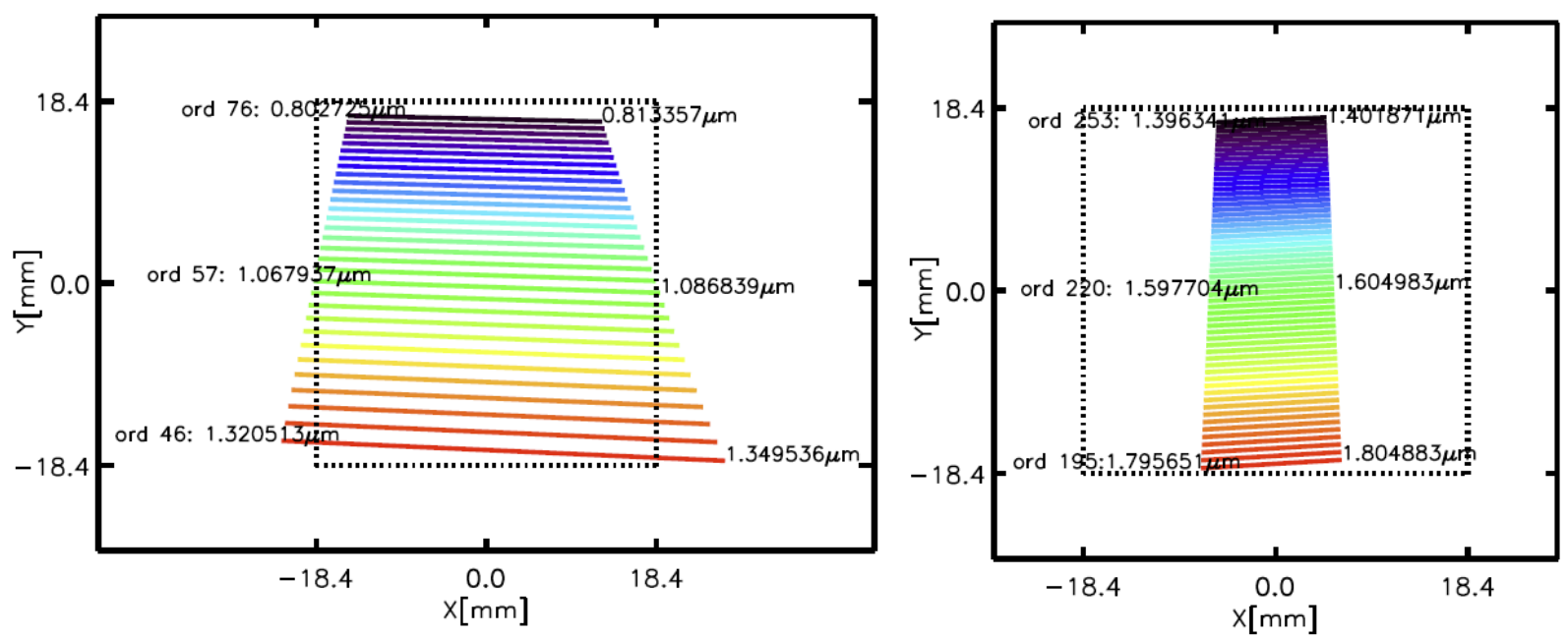

Figure 2.2. (Left) Spectral format of FIRST blue channel design on the $2 \mathrm{k} \times 2 \mathrm{k}$ H2RG IR array at $0.8-1.35 \mu \mathrm{m}$. The central box is the physical size of the detector. (Right). Spectral format of FIRST red channel design on the $2 \mathrm{k} \times 2 \mathrm{k}$ H2RG IR array at 1.4-1.8 $\mu \mathrm{m}$. The central box is the physical size of the detector.

describe the overall instrument system design, its predicted performance, current status, and a planned large-scale M dwarf planet survey.

\section{Description of the FIRST instrument}

FIRST is a new generation, fiber-fed, highly stable, cryogenic, NIR high resolution, crossdispersed echelle spectrograph operated in a vacuum chamber (Figure 2.1). It has two channels: a blue channel with a commercial R4 echelle to cover $0.8-1.35 \mu \mathrm{m}$ at $\mathrm{R}=60 \mathrm{~K}$ and a red channel with a UF made silicon immersion grating (SIG) as the main disperser to simultaneously cover $1.4-1.8 \mu \mathrm{m}$ at $\mathrm{R}=72 \mathrm{~K}$. The blue channel will be used for the M dwarf survey observations (science) while the red channel will be primarily used for the SIG high resolution spectroscopy technology demonstration. The spectra are recorded with an $\mathrm{H} 2 \mathrm{RG} 2 \mathrm{~K} \times 2 \mathrm{~K}$ array from Teledyne Technologies Inc with $2.57 \mu \mathrm{m}$ long wavelength cutoff. The detector is controlled by the standard ASIC card purchased from Teledyne. The entire instrument bench (1.0x0.5 meter in dimension) is installed inside a vacuum chamber. A 20-layer MALI thermal shield is mounted around the bench to largely insulate the bench from the chamber thermally.
Table 2.1. FIRST Instrument characteristics

\begin{tabular}{|l|l|}
\hline Wavelength region & $0.8-1.8 \mu \mathrm{m}$ \\
\hline Focal plane & $2048 \times 2048 \mathrm{H} 2 \mathrm{RG}$ \\
\hline Pixel size & $18 \mu \mathrm{m}$ \\
\hline Detector QE & $81 \% 0.6-1.0 \mu \mathrm{m}$ \\
& $90 \% 1.0-2.4 \mu \mathrm{m}$ \\
\hline Spectral resolution & $\mathrm{R}=60 \mathrm{~K}$ at $0.8-1.35 \mu \mathrm{m}$ (Blue) \\
& $\mathrm{R}=72 \mathrm{~K}$ at $1.4-1.8 \mu \mathrm{m}$ (Red) \\
\hline Dispersion per & $0.10 \AA$ 解 at $1.25 \mu \mathrm{m}$ (Blue) \\
pixel & $0.11 \AA /$ pix at $1.65 \mu \mathrm{m}$ (Red) \\
\hline Sampling & 2 pixels \\
\hline Fiber diameter & 1.9 arcsec $(75 \mu \mathrm{m})$ \\
\hline Spectral orders & $46-76(0.8-1.35 \mu \mathrm{m})$ (Blue) \\
& $195-253(1.4-1.8 \mu \mathrm{m})$ (Red) \\
\hline Single exposure & $0.52 \mu \mathrm{m}$ at $0.8-1.35$ um (Blue) \\
coverage & $0.4 \mu \mathrm{m}$ at $1.4-1.8 \mu \mathrm{m}$ (Red) \\
\hline Design type & White pupil + refractive camera \\
\hline Collimated beam & $50 \mathrm{~mm}$ in diameter \\
\hline Main dispersers & $\mathrm{R} 4,31.61 / \mathrm{mm}, 76^{\circ}$ blaze (Blue) \\
& SIG, $16.11 / \mathrm{mm}, 54.74^{\circ}$ blaze (Red) \\
\hline Cross-dispersers & $2401 / \mathrm{mm} 7.0^{\circ}$ blaze (Blue) \\
(VPH gratings) & $3101 / \mathrm{mm}, 14.0^{\circ}$ blaze (Red) \\
\hline Optical bench & $1.0 x 0.5 \times 0.4 \mathrm{~meter}$ \\
\hline
\end{tabular}


The optical bench is cooled with three cryotigers using standard NF-55 gas and operates at 193K to reduce the thermal background while the detector is cooled by one cryotiger with PT-13 gas and runs at $77 \mathrm{~K}$ to reduce its dark current. Small heaters and Lakeshore temperature sensors are placed on the bench and the detector assembly to allow the bench and detector temperature precisely controlled. The same temperature control mechanism adopted for the UF built optical spectrographs in 2008-2011, such as EXtremely high Precision ExtrasolaR planet Tracker (EXPERT), has demonstrated $\sim 4 \mathrm{mK}$ temperature long term stability over a year (Ge et al. 2010). The entire instrument system will be placed inside a temperature-controlled instrument room of the $2 \mathrm{~m}$ AST at Fairborn Observatory with yearly temperature variation within $2 \mathrm{~K}$ to further reduce the instrument long term temperature variation.

FIRST adopts a white pupil design (e.g., the design of HARPS (Pepe e al. 2002)), which enables a very compact instrument and allows effective management of scattered light. The details about FIRST optical design are reported in a separate SPIE paper (Zhao \& Ge, 2012, this proceedings). FIRST is fed with three $75 \mu \mathrm{m}$ diameter fibers (1.9 arcsec on sky, one science, one sky and one calibration) at $f / 4$. The science beam is sliced into two halves by a Mirror Image Slicer developed from the traditional BowenWalraven Image Slicer (Bowen, 1938) and passes through the spectrograph entrance slit. The FIRST blue channel covers 31 spectral orders (orders 46-76, see Figure 2.2) at 0.8-1.35 $\mu \mathrm{m}$ in a single exposure with 95\% wavelength coverage while the red channel completely covers 59 spectral orders (orders 195-253, see Figure 2.2) at $1.4-1.8 \mu \mathrm{m}$ in a single exposure. Two broad filters, one passing the blue channel beam and the other passing the red channel beam, on a motorized rotation stage are used to select an observation channel. Table 2.1 summarizes FIRST instrument parameters.

FIRST is calibrated with flatfielding, wavelength calibration, RV calibration, and flux calibration (required for some targets). Flatfielding is done with a tungsten continuum lamp. The wavelength calibration is done with an Ur/Ne emission lamp. In order to precisely calibrate instrument drift to reach high RV precision $(\sim 4 \mathrm{~m} / \mathrm{s})$, a separate $\mathrm{Ur} / \mathrm{Ne}$ spectrum is recorded along with a star spectrum at the same

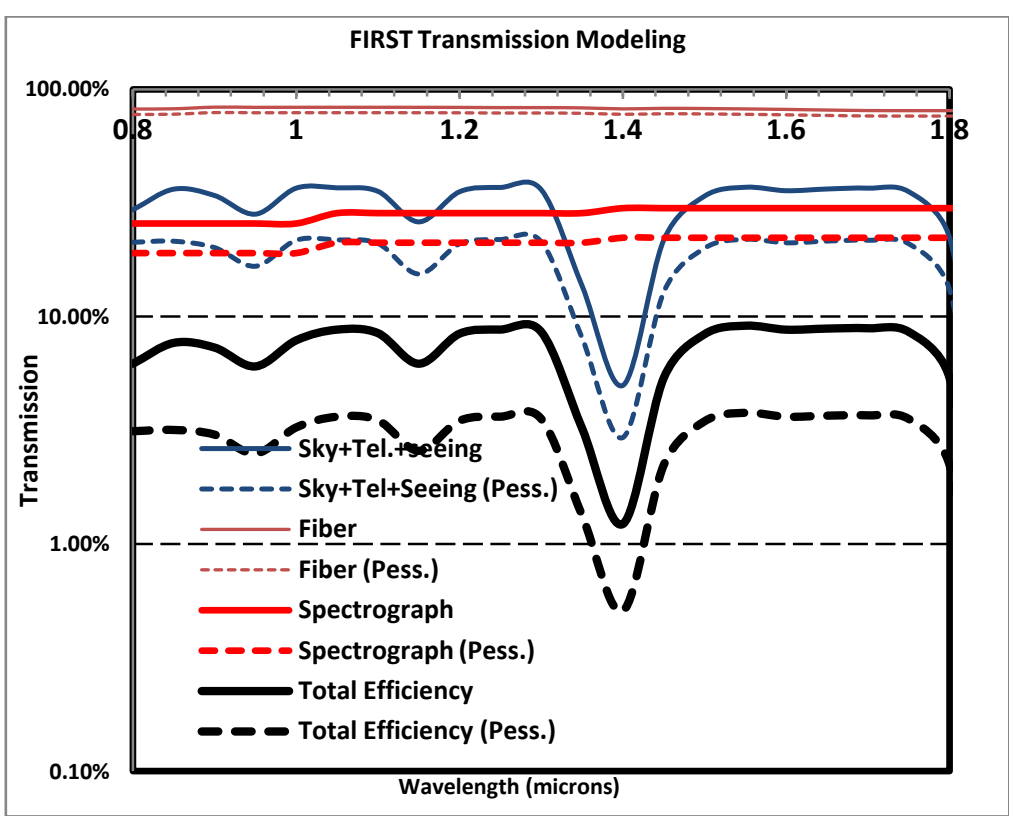

Figure 3.1. The FIRST instrument component transmission and overall throughput prediction (without including photon loss due to the planned fiber mode scrambling).

time. An Ur/Ne exposure is also taken through the science fiber before and after each star exposure to remove residual drifts not corrected by the simultaneous separate beam calibration. Since the Ur/Ne calibration lamp, like a ThAr lamp, has limited lifetime, frequent replacement of the Ur/Ne calibration lamp requires calibrating offsets for different lamps. This offset calibration is made by shining a second Ur/Ne lamp used occasionally (such as twice per month). Fibers (science and calibration fibers) will be mechanically mode scrambled to reduce illumination-induced variations in the RV measurements, which will also lead to reduction of the overall detection efficiency. 


\section{Predicted FIRST Performance}

\section{1. Detection efficiency}

Two cases have been considered for forecasting FIRST throughput: a baseline and a pessimistic case. The two cases have different telescope transmission, seeing coupling, fiber transmission, and spectrograph optics transmission (Table 3.1). Figure 3.1 shows transmission of various components for both cases and the predicted overall detection efficiency of FIRST in the $0.8-1.8 \mu \mathrm{m}$ region. The total detection efficiency at $0.8-1.8 \mu \mathrm{m}$ is $7-9 \%$ and $3-4 \%$ for the baseline and the pessimistic case, respectively.

\subsection{Detection Sensitivity}

The instrument detection efficiency in combination with photon noise, detector noises (dark current, $0.015 \mathrm{e}^{-}$ /s/pix and the single readout noise, $\left.13.4 \mathrm{e}^{-} / \mathrm{pix}\right)$, sky and telescope thermal background, instrument thermal background, and sky continuum emission (Maihara et al. 1993) is used to calculate detection sensitivity. Sky and telescope temperature of $300 \mathrm{~K}$ and $30 \%$ emissivity are used for thermal background calculations. Blackbody radiation of $193 \mathrm{~K}$ with $1.8 \mu \mathrm{m}$ long wavelength cutoff (no thermal radiation longer than $1.8 \mu \mathrm{m}$ reaches the detector) is used for calculating the instrument thermal background. $\mathrm{OH}$ emission lines are not included in the calculation. We plan to mask these $\mathrm{OH}$ lines out in the precision RV measurements. Figure 3.2 shows the detection sensitivity with FIRST in two instrument performance cases. In one hour, FIRST can achieve a SNR of $\sim 150$ per pixel (two pixel per resolution element in the dispersion direction) for a $\mathrm{I} \sim 10.8, \mathrm{~J} \sim 10.3$ or $\mathrm{H} \sim 9.7$ bright point source (such as M dwarfs), and a SNR of 10 per pixel for a $\mathrm{I} \sim 15.6, \mathrm{~J} \sim 15.2$, or $\mathrm{H} \sim 14.5$ faint point source.

\subsection{Doppler sensitivity}

High resolution synthetic stellar spectra, generated by the PHOENIX code (Hauschildt et al. 1999; Allard et al. 2001), were used in calculating photon-limited RV uncertainties with FIRST. FIRST instrument parameters and baseline instrument transmission were used in spectral synthesis. Table
Table 3.1. FIRST instrument transmission budget.

\begin{tabular}{|l|c|c|}
\hline Name & Baseline & Pessimistic \\
\hline Atmosphere & & \\
$0.9 \mu \mathrm{m}$ & 0.91 & 0.91 \\
$1.25 \mathrm{um}$ & 0.99 & 0.99 \\
$1.65 \mathrm{um}$ & 0.98 & 0.98 \\
\hline Telescope & 0.86 & 0.57 \\
\hline Seeing coupling & 0.43 & 0.39 \\
\hline Fiber & & \\
$0.9 \mu \mathrm{m}$ & 0.83 & 0.79 \\
1.25 um & 0.83 & 0.79 \\
1.65 um & 0.81 & 0.77 \\
\hline Spectrograph & & \\
$0.9 \mu \mathrm{m}$ & 0.32 & 0.23 \\
1.25 um & 0.32 & 0.23 \\
1.65 um & 0.33 & 0.25 \\
\hline Detector & & \\
$0.9 \mu \mathrm{m}$ & 0.81 & 0.81 \\
1.25 um & 0.90 & 0.90 \\
1.65 um & 0.90 & 0.90 \\
\hline & & \\
\hline Overall efficiency & & $\mathbf{0 . 0 3 0}$ \\
$\mathbf{0 . 9} \boldsymbol{\mu m}$ & $\mathbf{0 . 0 7}$ & $\mathbf{0 . 0 3 6}$ \\
$\mathbf{1 . 2 5}$ um & $\mathbf{0 . 0 9}$ & \\
$\mathbf{1 . 6 5}$ um & & \\
\hline
\end{tabular}


3.2 lists photon noise limited RV measurement errors in both channels. However, in the real RV measurements, the measured rms errors are usually larger than the photon noise limited errors. To predict the rms errors of RV measurements, we included measurement Table 3.2. Photon-limited RV measurement errors for $\mathrm{M}$ dwarfs with $\mathrm{S} / \mathrm{N}=150$ per pixel at $1.25 \mu \mathrm{m}$ in the blue channel and $1.65 \mu \mathrm{m}$ in the red channel.

\begin{tabular}{|l|l|l|l|l|l|l|}
\hline Vsini/Spec. type & M3V & \multicolumn{3}{l|}{ M6V } & M9V \\
\hline & Blue & Red & Blue & Red & Blue & Red \\
\hline $\mathbf{0 ~} \mathbf{~ k m} / \mathbf{s}$ & $2.5 \mathrm{~m} / \mathrm{s}$ & $2.3 \mathrm{~m} / \mathrm{s}$ & $2.1 \mathrm{~m} / \mathrm{s}$ & $1.8 \mathrm{~m} / \mathrm{s}$ & $1.6 \mathrm{~m} / \mathrm{s}$ & $1.3 \mathrm{~m} / \mathrm{s}$ \\
\hline $\mathbf{2 ~ k m} / \mathbf{s}$ & $2.7 \mathrm{~m} / \mathrm{s}$ & $2.5 \mathrm{~m} / \mathrm{s}$ & $2.2 \mathrm{~m} / \mathrm{s}$ & $1.9 \mathrm{~m} / \mathrm{s}$ & $1.7 \mathrm{~m} / \mathrm{s}$ & $1.4 \mathrm{~m} / \mathrm{s}$ \\
\hline $\mathbf{5 ~ k m} / \mathbf{s}$ & $3.6 \mathrm{~m} / \mathrm{s}$ & $3.3 \mathrm{~m} / \mathrm{s}$ & $3.0 \mathrm{~m} / \mathrm{s}$ & $2.6 \mathrm{~m} / \mathrm{s}$ & $2.2 \mathrm{~m} / \mathrm{s}$ & $1.9 \mathrm{~m} / \mathrm{s}$ \\
\hline
\end{tabular}

uncertainties from instrument RV calibration, contamination of telluric lines (absorption lines and $\mathrm{OH}$ emission lines), fiber illumination variations caused by the telescope guiding, tracking and seeing, instrument thermal drift and data pipeline. In the NIR region, telluric lines (especially absorption lines) are nearly everywhere. Modeling and removal of telluric contamination becomes a critical part of data processing to reach close to the photon noise limit (Blake et al. 2010; Wang et al. 2011). We calculated additional RV errors caused by masking out all of the telluric absorption lines at the $2 \%$ depth level and removing $\mathrm{OH}$ emission lines at the above $1 \%$ of the peak intensity level. We keep the RV uncertainty caused by the fiber Table 3.3. Error budget for a M6V star with Vsini $=2 \mathrm{~km} / \mathrm{s}$, $\mathrm{S} / \mathrm{N}=150$ per pixel at $1.25 \mu \mathrm{m}$.

\begin{tabular}{|l|l|l|}
\hline Error source/mode & Blue channel & Red channel \\
\hline Photon & $2.2 \mathrm{~m} / \mathrm{s}$ & $1.9 \mathrm{~m} / \mathrm{s}$ \\
\hline Calibration & $\sim 0.5 \mathrm{~m} / \mathrm{s}$ & $\sim 0.5 \mathrm{~m} / \mathrm{s}$ \\
\hline $\begin{array}{l}\text { Telluric absorption } \\
\text { removal (98\% level) }\end{array}$ & $2.0 \mathrm{~m} / \mathrm{s}$ & $1.5 \mathrm{~m} / \mathrm{s}$ \\
\hline $\begin{array}{l}\text { OH emission removal } \\
\text { (99\% level) }\end{array}$ & $1.5 \mathrm{~m} / \mathrm{s}$ & $2.9 \mathrm{~m} / \mathrm{s}$ \\
\hline $\begin{array}{l}\text { Fiber illumination } \\
\text { (guiding error) }\end{array}$ & $<0.5 \mathrm{~m} / \mathrm{s}$ & $<0.5 \mathrm{~m} / \mathrm{s}$ \\
\hline Thermal differential drift & $<1 \mathrm{~m} / \mathrm{s}$ & $<1 \mathrm{~m} / \mathrm{s}$ \\
\hline Data pipeline & $\sim 2.5 \mathrm{~m} / \mathrm{s}$ & $<2.1 \mathrm{~m} / \mathrm{s}$ \\
\hline Total error & $\sim 4.3 \mathrm{~m} / \mathrm{s}$ & $\sim 4.5 \mathrm{~m} / \mathrm{s}$ \\
\hline
\end{tabular}
illumination profile changes to within 0.5 $\mathrm{m} / \mathrm{s}$ by controlling the illumination change to within $1 \%$ through fiber mode scrambling in FIRST. We budget the RV measurement error caused by the thermal drift to within $1 \mathrm{~m} / \mathrm{s}$ by controlling the instrument long-term thermal stability to within $10 \mathrm{mK}$. Given many uncertainties in our RV extraction codes, we budget that the data pipeline can deliver 1.5 times the photon error. Table 3.3 summarizes the error budget in the RV measurements after including the above effects. We expect to reach $\sim 4-5 \mathrm{~m} / \mathrm{s}$ long term RV measurement errors with most of the $\mathrm{J}<9 \mathrm{M}$ dwarfs within 30 min exposures. Our $\mathrm{M}$ dwarf survey baseline design is based on this RV performance.

\section{Project Status}

The FIRST project was initiated in May 2009 when a high quality, large-format SIG with a $86 \times 50 \mathrm{~mm}^{2}$ etched grating area, $50 \times 50 \mathrm{~mm}^{2}$ entrance pupil and 54.7 degree blaze angle was available (Ge et al. 2006) and a $2 \mathrm{kx} 2 \mathrm{k}$ H2RG array and an ASIC control card were purchased. The FIRST team completed the instrument conceptual design and system requirement studies, including simulations and tradeoff studies, in January 2011. To date we have completed the optical design of the instrument and are finishing up the detailed design for mechanical components, cryostat, control and the detector package. All of the optics have been purchased. We have received a R4 echelle, both VPH grating cross-dispersers, three folding mirrors, band selection filters and a thermal blocking filter. Lab testing shows that performance of the commercial IR detector, R4 echelle, VPH gratings, filters and mirrors meet the requirements. The existing SIG was fully characterized in the lab in August to September 2011 and its performance meets the design requirements (Ge et al. 2012). Fabrication of the instrument mechanical components starts in early July, and subsystem component testing will be carried out in August and September 2012. 
The system integration and testing starts in late September 2012. Data pipeline development starts in June 2012 and an early version is available in December 2012 when the instrument system testing begins and the pipeline is used for processing lab testing data with calibration sources and sky. We expect to complete the lab testing and an instrument acceptance test by April 2013. We plan to deliver the instrument to the observatory in June 2013 and commission the instrument during the summer 2013.

Below summarizes key milestones of the project.

- Project kicks off September 2009

- Concept design review January 2011

- Preliminary design review October 2011

- Critical design review June 2012

- Acceptance test April 2013

- Commissioning June 2013

- First light September 2013

- M dwarf survey 2014-2017

\section{The FIRST NIR M Dwarf Planet Survey}

The FIRST NIR M dwarf planet survey is the first large-scale NIR high precision Doppler survey dedicated to detecting and characterizing planets around 215 nearby $M$ dwarfs with $\mathrm{J}<10$ with the $2 \mathrm{~m}$ AST at Fairborn Observatory. The primary science goal is to search for habitable Super-Earths around 101 low mass M4V and later type M dwarfs which have very close by habitable zones ( 1-75 daysor $\sim 0.01-0.3 \mathrm{AU}$ ) (Figure 5.1). With the forecast of an average of $\sim 4 \mathrm{~m} / \mathrm{s}$ baseline long term Doppler precision, FIRST can detect Super-Earths in the HZ around these late $M$ dwarfs. Any transiting SuperEarths in the HZ would be excellent candidates for follow-up observations with JWST to study their atmospheric compositions and possible biosignatures indicating life. The remaining $\sim 114$ survey targets are early type $\mathrm{M}$ dwarfs (M2V-M4V) with $\mathrm{V}>12$, none of which has yet been observed by any RV survey. The science goal is to conduct statistical studies of properties of low mass planets around these low mass stars along with our 101 high-priority late $\mathrm{M}$ dwarfs and constrain planet formation and evolution theories.

We take full advantage of the robotic nature of the AST $2 \mathrm{~m}$ telescope and its flexible queue observation schedule to design the survey with an optimized strategy, designing a schedule and cadence for the detection and characterization of planets, especially habitable planets around the low mass stars. For instance, each of the survey targets will be observed $\sim 24$ times randomly spread out over 100-day time

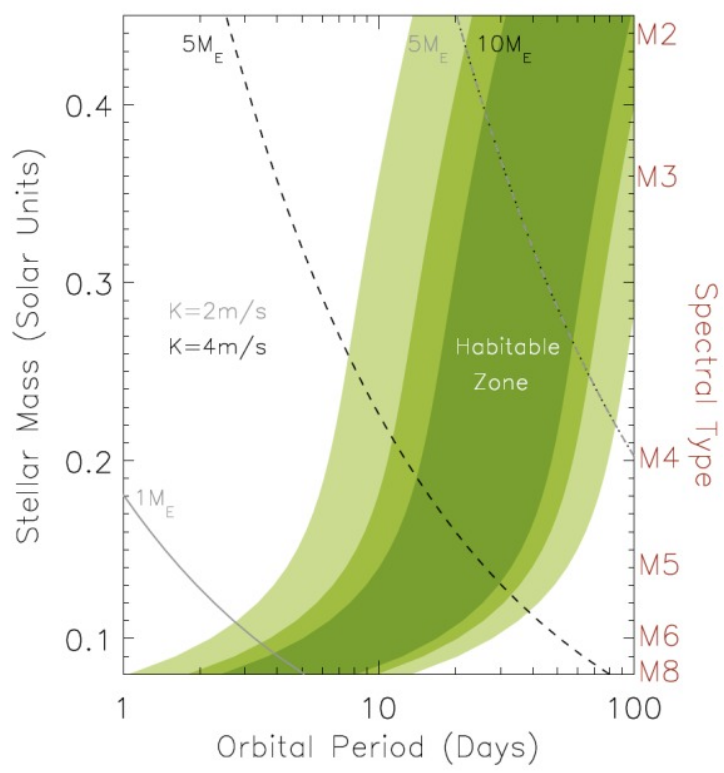

Figure 5.1. Late $\mathrm{M}$ dwarf orbit period vs. stellar host mass to be explored by the FIRST survey (Reid \& Hawley, 2000; Barnes et al 2012). The shaded regions are the HZ, with darker regions corresponding to less constraint for habitability (i.e., $0 \%, 50 \%$ to $100 \%$ cloud coverage, respectively). The dashed (dotted) curves show where planets induce a $4 \mathrm{~m} / \mathrm{s}(2 \mathrm{~m} / \mathrm{s})$ reflex velocity in the star, assuming a circular orbit. The left most curve is for a 1 Earth-mass planet, then 5, then 10. FIRST can detect planets to the left of the curve. window to minimize the time aliasing in detecting low mass planets, especially those in highly eccentric orbits. Figure 5.2 shows the predicted completeness 
from this baseline survey plan. This survey plan and strategy allow us to use both detections and nondetections from the survey to measure the survey completeness for statistical studies.

In order to minimize the risk associated with launching a large-scale RV survey with an untested new generation NIR high resolution spectrograph, we plan to first launch a pilot survey after FIRST on-sky performance (such as throughput, image quality, long term stability and Doppler precision) is fully measured and evaluated. The pilot survey will observe $50 \mathrm{M}$ dwarfs over 10 months in 2014-2015. Once the pilot survey establishes the statistical performance (especially RV precision and stability) of the instrument and data pipeline over a diverse set of survey targets (such as early to late $\mathrm{M}$ dwarfs with different rotational speeds and metallicities), the main survey will launch shortly after. This pilot survey will allow the team to tune hardware and the data pipeline ready for the main survey in 2015-2017.

\subsection{Survey Target Selection}

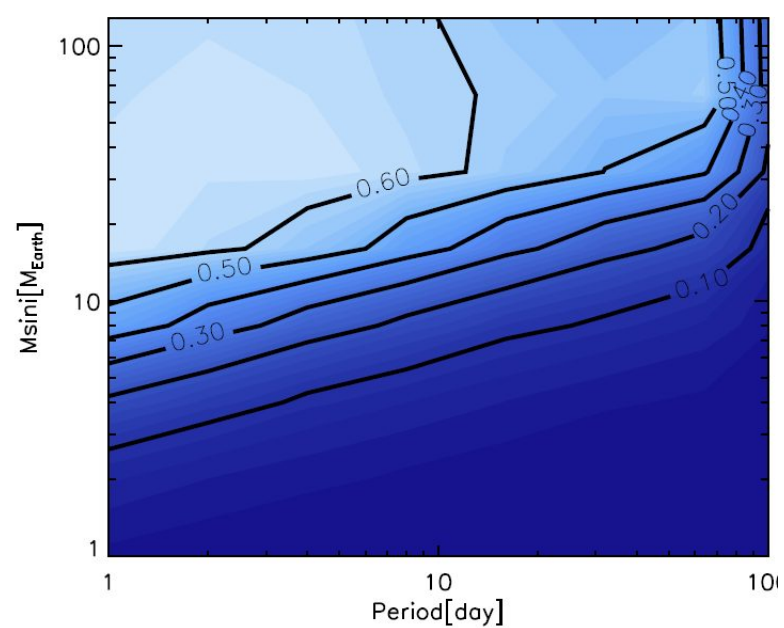

Figure 5.2. The predicted contours of the survey completeness based on the survey targets, observation strategy, cadence and baseline precision (see section 3.3).

Our targets were selected from the following catalogs: Gliese Catalog of Nearby Stars (Spring 1989); Gliese Catalog of Nearby Stars cross identified with 2MASS (Stauffer et al. 2010); ROSAT All-Sky Survey: Nearby Stars (Huensch et al. 1999); New M Dwarfs in the Solar Neighborhood (Riaz and Harvin 2006); and The Naval Observatory Merged Astrometric Dataset (NOMAD).

The selection was based on the following criteria:

- $\quad \mathrm{J}<10$ and dec $>-20$ (2062 stars selected)

- $\mathrm{Mv}<8.7$ and $\mathrm{V}-\mathrm{K}>3.5$

- Ratio between X ray luminosity and bolometric luminosity, $\mathrm{Rx}<-3.0$.

$215 \mathrm{M}$ dwarfs were selected with the above criteria (Note: before we launch the survey, we will use the 2MASS catalog to reject additional M dwarfs with a $\mathrm{J}<14$ stellar "companion" within 5 arcsec and replace them with slightly fainter $\mathrm{M}$ dwarfs. During the survey, we will reject spectroscopic binaries after $\quad 3 \quad \mathrm{RV}$ measurements from our targets and replace them with new survey targets). Based on the empirical equation of rotation velocity vs. $\mathrm{Rx}$ in Kiraga et al. 2007, we expect $87 \%$ of our $\mathrm{M}$
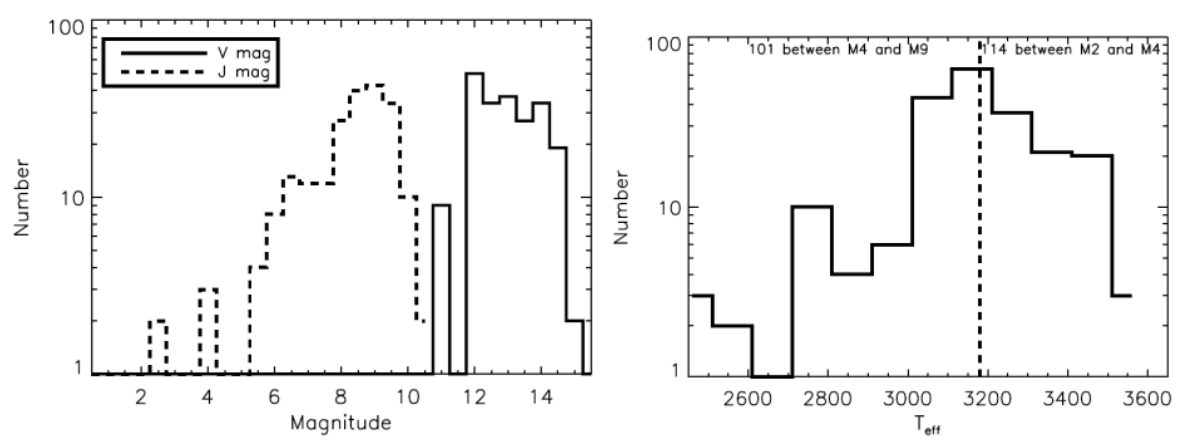

Figure 5.3. (Left). V and J magnitude distribution of selected stars for the FIRST M dwarf survey. (Right). $\mathrm{T}_{\text {eff }}$ distribution of selected $\mathrm{M}$ dwarfs. dwarfs with rotational velocity less than $5 \mathrm{~km} / \mathrm{s}$. Therefore, most of them are inactive stars, which can help to minimize the RV jitters caused by stellar activities although the jitter level is significantly reduced in NIR (e.g., Raines et al. 2010; Ma \& Ge 2012). Figure 5.3 shows the number distribution in the J and V 
bands of the FIRST M dwarf survey targets and the effective temperature distribution of the survey targets.

\subsection{Predicted Planet Yield}

Figure 5.4 shows predicted rms RV measurement errors of our survey targets based on 30 min exposure on targets (the baseline and pessimistic cases). The baseline measurement uncertainties include a combination of 1.5 times photon noise (including the pipeline error), calibration $(0.5 \mathrm{~m} / \mathrm{s})$ and telluric line contamination $(2.0 \mathrm{~m} / \mathrm{s})$ while the pessimistic measurement uncertainties include a combination of 3 times photon noise (including the data pipeline error and the pessimistic performance of the instrument total detection efficiency of $\sim 4 \%$, see section 3.1$)$, calibration $(0.5 \mathrm{~m} / \mathrm{s})$, telluric line contamination $(2.0 \mathrm{~m} / \mathrm{s})$, and a $3 \mathrm{~m} / \mathrm{s}$ long term systematic error are applied to all survey targets. The photon error is calculated from the formula derived by Bouchy et al. (2001) using the following instrument parameters: $\mathrm{R}=60,000$, $95 \%$ coverage of $0.8-1.35 \mu \mathrm{m}$, and $8 \%$ total detection efficiency (see 3.1 for details). The calibration error is calculated from an emission spectrum of a Ur/Ne lamp (Redman et al. 2011), which is used to calibrate the instrument drift. Spectral regions with telluric absorption more than $2 \%$ are masked out in RV calculation, which contributes about $2.0 \mathrm{~m} / \mathrm{s}$ measurement uncertainties. The pipeline produces uncertainties on the order of $\sim 2.5 \mathrm{~m} / \mathrm{s}$ and $\sim 5 \mathrm{~m} / \mathrm{s}$ for a typical survey star (J 9) for the baseline and pessimistic cases, respectively. Simulations did not include other measurement errors, such as the instrument differential drift, the guiding error, and $\mathrm{OH}$ contamination error, which are relatively small and do not affect the simulation results (see Table 3.3 for detailed error budget).

Based on the RV measurement uncertainties in both baseline and pessimistic cases and planned survey strategy, in terms of schedule and cadence, we conducted survey simulations to derive survey sensitivity and completeness (Figure 5.2 shows the baseline result). Once RV precision and stellar mass is known, we generate a detectability plot in the mass-period space. More specifically, for a given planet mass and orbital period, we generate a RV curve of 100 days from which $24 \mathrm{RV}$ points are randomly drawn to form an RV data set. The data set is then analyzed by a detection code based on a periodogram. If the peak of the periodogram agrees with the input period and the FAP is less than $1 / 1000$, then we mark it as a "detection". This test is repeated 100 times for each given planet mass and period. We repeat this detectability/completeness plot for each star. The survey completeness plot (Figure 5.2) is the sum of completeness plots of all selected stars.

Our full survey baseline is expected to detect $\sim 10$ Super-Earths, $\sim 18$ intermediate-mass planets and 2 giant planets within 100 day periods, respectively, assuming the planet yield is $\eta=35 \%, 18 \%$ and $1.5 \%$ (Bonfils et al. 2011) are the frequencies of SuperEarths (less than 10 Earth masses), intermediate-mass planets (10-100 Earth masses) and giant planets (above 100-1000 Earth masses) around M dwarfs with 1-100 day period, respectively, while the pilot survey is expected to detect $\sim 11$ new planets including $\sim 5$

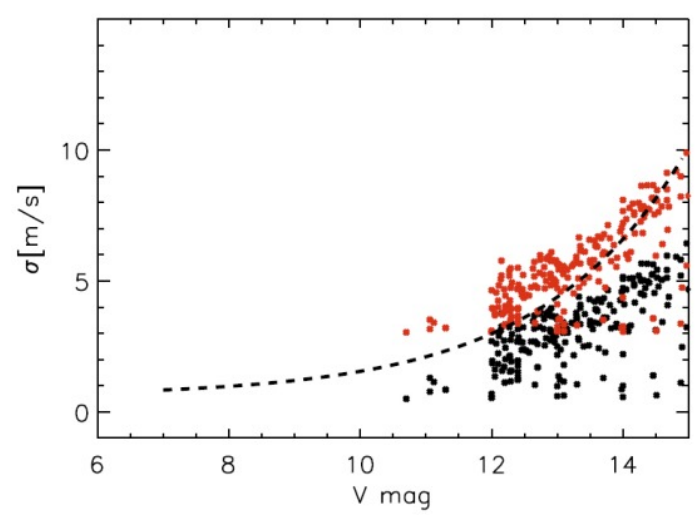

Figure 5.4. Predicted RV measurement precision of our survey targets in the baseline case (black dots) and pessimistic case (red dots). Dashed line is used to guide an eye which represents the best RV precision achieved by Bonfils et al. 2011. The project FIRST baseline performance is about a factor of two times better than the HARPS M dwarf survey due to the significant photon gain in the $\mathrm{Y}+\mathrm{J}$ band of late $\mathrm{M}$ dwarfs over the HARPS $\mathrm{V}+\mathrm{R}$ band.

Super-Earths. About half of the Super-Earths ( $\sim 5$ Super-Earths) in the full survey will be in their HZ (33\% without cloud coverage and $73 \%$ with full cloud coverage) and one of them may be a transiting planet. Our pessimistic survey case is expected to detect $\sim 6$ Super-Earths, $\sim 16$ intermediate mass planets and $\sim 2$ giant planets. Additional planets (such as $\sim 3$ Super-Earths and $\sim 2$ intermediate mass planets) may 
be confirmed with additional follow-up observations of candidates with $\sim 0.5 \%$ FAP. Our baseline detections would nearly double planet detections over the detections from the HARPS M dwarf survey (Bonfils et al. 2011), substantially increasing the power for statistical study of planet occurrence and properties and constraining on planet formation models and physical conditions.

\section{Conclusions}

FIRST is the first high resolution NIR cross-dispersed echelle spectrograph designed and optimized for high precision RV measurements of $\mathrm{M}$ dwarfs. It can offer a nearly complete wavelength coverage with $\mathrm{R}=60,000$ at $0.8-1.35 \mu \mathrm{m}$ and a complete wavelength coverage with $\mathrm{R}=72,000$ at $1.4-1.8 \mu \mathrm{m}$ in a single exposure with a $2 \mathrm{kx} 2 \mathrm{k} \mathrm{H} 2 \mathrm{RG}$ IR array thanks to the use of an extremely high dispersion R1.4 (a blaze angle of 54.7 degree) SIG (1.4-1.8 $\mu \mathrm{m}$ ) and a commercial R4 echelle (a blaze angle of 76 degree) with a $50 \mathrm{~mm}$ diameter pupil in combination with a Bowen-Walraven-type Image Slicer. With vacuum operation and accurate temperature control, it can reach $4 \mathrm{~m} / \mathrm{s}$ long term RV precision with $\mathrm{J}<9 \mathrm{M}$ dwarfs within 30 min exposures and a possible better precision if the telluric lines can be modeled and removed at better than the $2 \%$ depth level. FIRST is scheduled to be commissioned at the AST in summer 2013. The pilot survey of $50 \mathrm{M}$ dwarfs will be carried out in 2014-2015, followed with the full survey of $165 \mathrm{M}$ dwarfs in 2015-2017. Based on the baseline performance of the instrument, we expect to detect $~ 30$ exoplanets, including 10 Super Earths, within 100 day periods. About half of the Super-Earths are in their habitable zones and one of them may be a transiting planet. We will look for habitable Super-Earths around the late M dwarfs and also to identify transiting systems for follow-up observations with JWST to measure the planetary atmospheric compositions and study their habitability. We will also detect and characterize a large number of planets around $\mathrm{M}$ dwarfs to understand the statistics of planet populations around these low mass stars and constrain planet formation and evolution models. As a high resolution NIR spectrograph, FIRST can also be used for observing young stars, transiting planets, young stellar objects (YSOs), magnetic fields, binaries, brown dwarfs (BDs), ISM and stars.

\section{ACKNOWLEDGEMENTS}

We acknowledge the support from DoD Cooperative Agreement W911NF-09-2-0017, Dharma Endowment Foundation, NSF with grant NSF AST-0705139, NASA with grants NNX07AP14G and NNG05GR41G and the University of Florida. 


\section{References:}

[1]Allard, F., et al. “,” ApJ, 556, 357 (2001)

[2] Barnes, R. K. Mullins, C. Goldblatt, V. S. Meadows, J. F. Kasting, and R. Heller, ArXiv e-prints, March (2012)

[3] Bean, J. et al. “,” ApJ, 713, 410 (2010)

[4] Blake, C.H. et al. “,”ApJ, 723, 684 (2010)

[5] Bowen, I.S. “,” ApJ, 88, 113(1938)

[6] Bonfils, X. et al.,"," arXiv1111.5019B (2011)

[7] Borucki, W.J., et al., "," ApJ, 736, 19(2011)

[8] Bouchy, F., Pepe, F., \& Queloz, D. “,” A\&A, 374, 733(2001)

[9] Eaton, J.A., \& Williamson, M.H. "," PASP, 119, 886(2007)

[10] Endl, M. et al. “,”AJ, 131, 3131(2006)

[11] Ge, J., McDavitt, D., Zhao B., \& Miller, S. “,” Proc. SPIE, 6273, 72(2006)

[12] Ge, J. et al. "," Proc. SPIE, 7735, 16(2010)

[13] Ge, J. et al. “,” Proc. SPIE, 8446, in press (2012)

[14] Hauschildt, P. H., Allard, F., \& Baron, E. “,” ApJ, 512, 377(1999)

[15] Hunsch, M. et al. “,” A\&AS, 135, 319(1999)

[16] Johnson, J. et al. “,” ApJ, 670, 833(2007)

[17] Kiraga, M., \& Stepien, K. “,”ACA, 57, 149(2007)

[18] Lepine, S. \& Shara, M., “,”AJ, 129, 1483(2005)

[19] Ma, B., \& Ge, J., “,” ApJ, 750, 172(2012)

[20] Maihara, T. et al. “,” PASP, 105, 940(1993)

[21] Mayor, M. et al. “,”A\&A, 507, 487(2009)

[22] Pepe, F., et al. “,” The Messenger (ISSN 0722-6691), No. 110, p. 9 - 14(2002)

[23] Pepe, F. et al. “,"A\&A, 534, 58(2011)

[24] Reid, I.N., \& Hawley, "," S.LNew light on dark stars. Red dwarfs, low-mass stars, brown dwarfs, by

Reid, I. N.; Hawley, S. L.. Springer, London (UK), XXV + 470 p., ISBN 1-85233-100-3, Published in association with Praxis Publishing, Chichester (UK)(2000)

[25] Reiners, A., Bean, J., Huber, K., Dreizler, S., Seifahrt, A., \& Czesla, S. “,” ApJ, 710, 432(2010)

[26] Redman, S.L., Lawler, J.E., Nave, G., Ramsey, L.W., \& Mahadevan, S. “,” ApJS, 195, 24(2011)

[27] Reid, N. \& Gizis, J. “,”AJ, 113, 2246(1997)

[28] Riaz, B. et al. “,”AJ, 132, 866(2006)

[29] Stauffer, J. et al. “,’PASP, 122, 885(2010)

[30] Wang, J. Ge, J., “,”ApJ (submitted, arXiv:1107.4720) (2011)

[31] Wang, J., Ge, J., Peng, J., \& Zhao, B. “,”ApJ , 738, 132(2011)

[32] Zhao, B., \& Ge, J., “,” Proc. SPIE, 8446, in press (2012) 